\title{
Hearing screening procedures and protocols in use at immunisation clinics in South Africa
}

\begin{tabular}{|c|c|}
\hline \multicolumn{2}{|c|}{$\begin{array}{l}\text { Authors: } \\
\text { Luisa Petrocchi-Bartal }^{1} \\
\text { Katijah Khoza-Shangase }^{1}\end{array}$} \\
\hline $\begin{array}{l}\text { Affiliations: } \\
{ }^{1} \text { Department } \\
\text { Pathology an } \\
\text { School of Hur } \\
\text { Community D } \\
\text { University of } \\
\text { Witwatersran }\end{array}$ & $\begin{array}{l}\text { f Speech } \\
\text { Audiology, } \\
\text { han and } \\
\text { evelopment, } \\
\text { he } \\
\text { d, South Africa }\end{array}$ \\
\hline \multicolumn{2}{|c|}{$\begin{array}{l}\text { Correspondence to: } \\
\text { Katijah Khoza-Shangase }\end{array}$} \\
\hline \multicolumn{2}{|c|}{$\begin{array}{l}\text { Email: } \\
\text { katijah.khoza@wits.ac.za }\end{array}$} \\
\hline \multicolumn{2}{|c|}{$\begin{array}{l}\text { Postal address: } \\
\text { Box } 57 \text {, University of the } \\
\text { Witwatersrand 2050, } \\
\text { South Africa }\end{array}$} \\
\hline \multicolumn{2}{|c|}{$\begin{array}{l}\text { Dates: } \\
\text { Received: } 26 \text { Feb. } 2014 \\
\text { Accepted: } 10 \text { Aug. } 2014 \\
\text { Published: } 03 \text { Dec. } 2014\end{array}$} \\
\hline \multicolumn{2}{|c|}{$\begin{array}{l}\text { How to cite this article: } \\
\text { Petrocchi-Bartal, L., \& } \\
\text { Khoza-Shangase, K. (2014). } \\
\text { Hearing screening procedures } \\
\text { and protocols in use at } \\
\text { immunisation clinics in South } \\
\text { Africa. South African Journal } \\
\text { of Communication Disorders, } \\
61(1), \text { Art. \#66, } 9 \text { pages. } \\
\text { http://dx.doi.org/10.4102/ } \\
\text { sajcd.v61i1.66 }\end{array}$} \\
\hline \multicolumn{2}{|c|}{$\begin{array}{l}\text { Copyright: } \\
\text { (C) 2014. The Authors. } \\
\text { Licensee: AOSIS } \\
\text { OpenJournals. This } \\
\text { work is licensed under } \\
\text { the Creative Commons } \\
\text { Attribution License. }\end{array}$} \\
\hline \multicolumn{2}{|l|}{ Read online: } \\
\hline 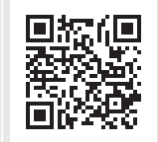 & $\begin{array}{l}\text { Scan this QR } \\
\text { code with your } \\
\text { smart phone or } \\
\text { mobile device } \\
\text { to read online. }\end{array}$ \\
\hline
\end{tabular}

Background: There exists a need for context-relevant research aimed at facilitating the efficacious provision of early hearing detection and intervention services in South Africa.

Objectives: This study aimed to determine the hearing screening procedures and protocols as well as referral protocols in use at maternal child woman's health (MCWH) immunisation clinics in South Africa.

Method: Thirty primary health care immunisation clinic managers or acting managers were interviewed in two South African sample groups. An exploratory, non-experimental, qualitative research design was employed incorporating both quantitative and qualitative information. An interview using a questionnaire was administered with all participants. The questionnaire encompassed areas such as work contexts, hearing screening contexts and information management systems, as well as quality control measures in place at these clinics. Content analysis was then used to code emergent themes into specific categories. Frequency calculations of these themes were calculated and results described qualitatively.

Results: No primary health care (PHC) clinics placed within the identified sites provided formalised new-born/infant hearing screening and none of these facilities had equipment to do so. Most sites attributed the lack of formalised hearing screening to budgetary and human resource issues, staff training in particular. Non-formalised hearing screening protocols in place demonstrated inconsistencies in application across districts and none complied with Health Professions Council of South Africa (HPCSA) clinic guidelines or any international guidelines.

Conclusion: Results from the current study have assisted in identifying procedural and logistical assets and barriers to implementation of HPCSA clinic guidelines for early hearing detection and intervention (EHDI) at immunisation clinics in South Africa.

\section{Introduction}

Literature has convincingly illustrated the importance of hearing function in childhood development as a fact that is evidence supported. A crucial interdependence between a child's ability to hear and his language development has been well documented (Northern \& Downs, 2002). Infancy has been reported as the critical period in which language learning is crucial to prevent life-long disadvantages (Moeller, 2000; Northern \& Downs, 1991; Yoshinaga-Itano, 2004). Language learning can be significantly affected by hearing impairment. The negative influence hearing impairment usually exerts on a child's development may be pervasive, affecting areas such as cognition (Olusanya, 2005), language, educational, social and emotional competence (Northern \& Downs, 1991), literacy development (DesJardin, Ambrose, Martinez, \& Eisenberg, 2009), as well as the individual's vocational and thus financial outcomes (Olusanya, 2000; Olusanya, Ruben \& Parving, 2006).

Although the effects of hearing loss initially impact at the level of the individual once the everyday communication process is hindered (Northern \& Downs, 1991), these subsequently develop into a societal economic long-term burden if the issue of reduced hearing ability is not addressed as soon as it can possibly be detected (Health Professions Council of South Africa [HPCSA], 2007; Joint Committee on Infant Hearing Screening [JCIHS], 2007; Moeller, 2000; Yoshinaga-Itano, 2004). This extended societal burden and decreased quality of life for the individual can be prevented and/or aleviated by the ability of a hearing screening programme to effectively identify infants and implement intervention at the earliest opportunity (Hutt \& Rhodes, 2008).

Internationally, early hearing detection and intervention (EHDI) has been accepted as a measure of child health care best practice (Olusanya, 2005); with universal new-born hearing screening (UNHS) being the most preferred model. This has been highlighted internationally 
as the preferred approach for hearing health care in private and public sectors of health care management (JCIH, 2000; Olusanya, Luxon \& Wirz, 2005); where adherence to the early intervention principles as they pertain to Audiology is the goal. These principles include a recommendation for diagnosis of hearing impairment with early intervention services implemented by six months of age internationally (JCIHS, 2007), and by a maximum of eight months of age in South Africa (HPCSA, 2007).

In the United States of America (USA) (Mehl \& Thomson, 2002; Theunissen \& Swanepoel, 2008) and many other developed countries (JCIHS, 2007); the feasibility and benefits of EHDI programmes are well documented; with some developing countries (Yee-Arellano, Leal-Garza \& Pauli-Muller, 2006; Olusanya, 2007) having in fact promulgated hearing screening policies based on JCIH principles (Yee-Arellano, Leal-Garza, \& Pauli-Muller, 2006). However, most developing world contexts have not formally recognised the advantages UNHS presents and have consequently not legislated any hearing screening protocols (Olusanya, 2007).

Reasons cited for this lack of EHDI implementation in most developing country contexts are unclear and complex. Nonetheless, one can assume that additional factors often endemic to regions like sub-Saharan Africa, including poverty (Olusanya, 2000) and the burden of life-threatening diseases such as human immunodeficiency virus and/or acquired immune deficiency syndrome (HIV and/or AIDS) and tuberculosis (Streefland, 2005) have a measurable influence. Arguably, in these contexts, hearing impairment may be low on the priority list and presents with comparatively less urgency; rendering implementation of EHDI to be less important (Olusanya, 2005).

It is well recognised that even within developed world contexts, EHDI's sustainability is challenged when implemented without the weighting of contextual relevance (Olusanya, 2005) and without appropriate cohesion and collaboration between relevant stakeholders (White, 2006). Therefore, EHDI programme efficacy and sustainability is reported to only be achievable if the setting in which it is to be implemented is considered specifically (Olusanya, 2005). This is further supported by the acceptable tenet that EHDI programmes must be contextually relevant; hence even more crucial in the developing world, where the level of additional difficulties these regions face are considerable compared to developed world environments (Swanepoel, Louw \& Hugo, 2007).

Given South Africa's inherent cultural and linguistic diversity (World Bank, 2009), the HPCSA (2007) has recognised the need for contextual and cultural congruency for EHDI to be effective within the South African milieu. The HPCSA (2007) has in fact provided concrete guidelines, benchmarks and quality indicators for different EHDI contexts in their position statement (HPCSA, 2007). Furthermore, in an effort to continuously improve EHDI services; this council has highlighted the importance of evidence-based assessments of these guidelines and benchmarks within these specifically defined contexts. HPCSA (2007) Clinic screening benchmarks and quality indicators include:

- Ninety-five percent of infants through their attendance of their six week immunisation should be screened after six months of initiation of the clinic screening programme, as reflected in the quality indicator of actual percentage of infants screened at their six week immunisation visit as well as the percentage of those screened at the age of six weeks.

- A less than 5\% referral rate for audiological and medical assessment within one year of initiation of the programme.

- Efforts to follow-up on a minimum of $95 \%$ referrals for a rescreen post the initial screen to be documented by the audiologist managing the EHDI programme, with adequate consideration of influencing factors such as (1) a lack of demographic information, (2) changes in contact details or addresses, (3) facility access, and (4) personal limitations including poverty. A return rate of $70 \%$ or more is considered ideal.

Further overall quality indicators include calculation and documentation of:

- Percentage of bilateral refers for a rescreen/diagnostic evaluation targeting permanent bilateral hearing loss of a minimum of $40 \mathrm{~dB}$ averaged across $500 \mathrm{~Hz}, 1,2$ and $4 \mathrm{kHz}$.

- Percentage of unilateral refers requiring rescreens in six to nine months.

- Percentage of refers who return for follow-up services, be they rescreens or diagnostic services.

- Percentage of rescreen refers who are referred for audiologic and/or medical evaluation.

- Percentage of families who refuse screening.

- Percentage of caregivers who report a positive attitude towards the screening programme post the initial screen (HPCSA, 2007).

Literature review within sub-Saharan Africa indicates paucity of contextually relevant evidence. It is only countries such as Nigeria and South Africa that have published researched aspects of EHDI as they apply in varying contexts within this part of the developing world (Olusanya \& Okolo, 2006). Literature available in these countries reveals a nonsystematic, piecemeal approach to evidence gathering, with a need for comprehensive and systematic research in all aspects of EHDI. To date, EHDI within the South African context can be argued to be under preliminary investigation, with only several published results available. Three of these investigations are relatively recent research efforts, with earlier publications spanning the years 2006-2010. Within the private sector specifically, Swanepoel, Ebrahim, Joseph, and Friedland (2007) investigated new-born hearing screening in a South African private health care hospital; and Meyer, Swanepoel, Le Roux and Van der Linde (2012) explored early detection of infant hearing loss in the private health care sector of South Africa; while Scheepers, Swanepoel and Le Roux (2014) interrogated the reasons why parents refuse hearing screening and default on follow-up rescreening 
in South Africa. The four located in different aspects of the government sector include an investigation by Swanepoel, Hugo, and Louw (2006) on infant hearing screening at immunisation clinics in South Africa; a study by Theunissen and Swanepoel (2008) on EHDI services in the public health sector in South Africa; a retrospective review by Kanji, KhozaShangase and Ballot (2010) on hearing screening follow-up return rate in a very low birth weight project; and an analysis of the efficacy of a community-based hearing screening program utilising existing clinic personnel in Western Cape, South Africa by Friderichs, Swanepoel and Hall (2012). This relatively small number of published data reflects a lack of actualised hearing screening in different contexts of community practice. These studies, in fact, directly or by implication, recommend for intensified focus in researching the field of early hearing detection and intervention in the South African context so as to formulate appropriate hearing screening protocols based on scientific evidence.

Assessment of the viability of a hearing screening programme in its original and natural environment is a vital determinant regarding the feasibility of HPCSA EHDI guidelines and the associated UNHS benchmarks in the South African context. It is within this position that the current study is located. The current study is located within the government sector where it has been observed that hearing screening, nationally, is not taking place as a matter of routine according to the protocols delineated (Theunissen \& Swanepoel, 2008). It is within the public sector where clinical services are most commonly accessed by the majority of South Africans, introducing another reason why the current study within the public sector is relevant. If the South African 1997 White Paper on the Integrated National Disability Strategy, which advocates equal opportunity for all, is to be actualised for those challenged by impaired hearing ability (Office of the Deputy President T.M. Mbeki, 1997; Theunissen \& Swanepoel, 2008); efforts to improve evidence-based practice need to be increased.

Specifically, if inroads are to be made towards actualising the ultimate aim of reducing disability through application of a contextually specific UNHS programme, with appropriate combination of theoretical and logistical constructs, key issues within the specific screening context must be identified and explored (JCIH, 2000; JCIHS, 2007; HPCSA, 2007). The current study aimed to establish the hearing screening procedures and protocols in use at Mother, Child and Woman Health (MCWH) immunisation clinics in NorthWest and Gauteng provinces; as part of a bigger study that explored the feasibility of implementing the HPCSA's (2007) guidelines for clinic based hearing screening in the South African primary health care clinic setting.

\section{Methodology}

The aim of the current study was to establish the hearing screening procedures and protocols as well as referrals in use at MCWH immunisation clinics in the North-West and Gauteng provinces in South Africa.

\section{The sample}

Thirty primary health care nurses were selected as participants for the current study and were recruited from primary health care immunisation clinics located within the district MCWH Cluster construct, where all services rendered were, and are at a health care level one. Within the MCWH cluster, community nurses and lay volunteers provide services including the implementation of immunisation programmes, where yield (actual immunisations generated out of the possible population in the area) is reported to be $91.8 \%$ in Gauteng and $71.4 \%$ in the North-West (Day \& Gray, 2008). More specifically, these mobile and fixed clinics may be described as predominantly outpatient facilities, which incorporate the specific targeted population of infants requiring immunisations at 6,10 and 14 weeks; 9 and 18 months, and 5 years (Office of the President, K. Motlanthe, 2009). For the purposes of the current study, in order to facilitate improved control over extraneous variables, as recommended by Maxwell and Satake (2006), only nurses stationed at fixed non-mobile clinics were included.

Participants were drawn from two provinces in South Africa; namely Gauteng and North-West provinces. These two provinces are considered to generally differ demographically in terms of socio-economic development based on the deprivation index as estimated from the Community Survey (Statistics South Africa, 2007), with the North-West population group considered to be more disadvantaged than that of Gauteng province (Day \& Gray, 2008). Socio-economic indicators are considered to measure some of the most important health determinants within a primary health care approach (Day \& Gray, 2008). The overall differences between North-West and Gauteng provinces were aimed at facilitating a rural-urban divide, and these were thought to be useful for assessing hearing health care outcome determinants within the context of this study by the current researcher.

\section{Sample size, distribution and sampling procedure}

Thirty primary health care clinic nurses in charge of 30 primary health care immunisation clinics within the NorthWest and Gauteng provinces (15 per province) were recruited according to accessibility and according to the district demographic classification (Day \& Gray, 2008). This nonprobability purposive participant recruitment strategy was adopted to ensure that the deprivation index difference in profile between the two provinces was maintained. These nurses were either officially within the position of Operations Manager or Acting Operations Manager or heading the clinic at that time if the incumbent was unavailable for the interview. The interview location (for all interviews including those conducted for the pilot project) was the participant's immunisation clinic.

Within this selected demographic location, convenience sampling of appropriate primary health care clinics ensued, where the offering of immunisations to infants at 6, 10 and 14 weeks was a prerequisite (Maxwell \& Satake, 2006). 


\section{Participants}

\section{Participant selection - inclusion or exclusion criteria}

For participants to be included in the current study, the following criteria had to be met:

1. The health care nurse interviewed was required to be in charge of the individual clinic's overall functioning. As the study was located within the context of the Department of Health (DoH)'s MCWH Cluster, specifically the immunisation scheduled programme, the health care nurse in charge of the individual clinic's overall functioning (or in the position of acting clinic manager), was recruited for the interview process. A main reason for selecting the clinic manager as interviewee was that being at the helm implies insight as to the detailed workings of the particular clinic in question (S. Mkoka, personal comm., August 11, 2009).

2. Health care nurses interviewed were required to be located within Gauteng and North-West provinces in designated proportions as defined under sample selection.

3. Each participant needed to be conversant in English as the interviews were conducted in English.

\section{Test p-Protocol \\ Material and apparatus}

The following materials were used to obtain data from the current study.

\section{Participant information sheets and consent forms}

An information sheet(s) describing the purpose of the study and the process involved was presented to each participant prior to initiation of the interview:

1. Interview schedule: The interview schedule and questionnaire used were structured where most questions presented were factual and closed-ended. These were supplemented by several open-ended questions to enable documentation of free thought processes which the researcher transcribed verbatim. The questionnaire was adapted from a self-administered questionnaire previously used in a study conducted in a collaborative effort by EHDI South Africa, the Centre for Deaf Studies and Deaf Education, the University of Pretoria and the University of the Witwatersrand (Theunissen \& Swanepoel, 2008). The interview schedule used in the face-to face interviews, consisted of the following sections:

- demographic information

- work context

- hearing screening context

- information management and quality control.

2. Tape recorder: A digital tape recorder (Sony ICD-UX81F) was used to record interviews to increase the accuracy of the responses documented by cross-checking the verbatim transcription of responses already conducted during the interview. Using a tape recorder to verify transcriptions improves the quality of the data collection and reliability and validity of the transcription process (Maxwell \& Satake, 2006).

\section{Procedures}

Prior to the study being conducted, permission was sought from the University of the Witwatersrand Human Ethics Committee (Medical), and approval to conduct the research (clearance certificate number M091040) was obtained. This approval, together with a detailed research proposal was then submitted to the Gauteng Province and North-West Province Directors: Policy, Planning and Research divisions. Permission was obtained from the respective province directors. Additional levels of written permission, as well as verbal permission were obtained from the specific sub-district divisions to ensure ease of access to the respective clinics. Thereafter, permission was obtained from individual immunisation clinic managers or their assigned deputy so as to ensure the participants' autonomy. Formal informed consent and participant agreement to partake in the research process was provided prior to the commencement of each interview where permission to proceed was granted by the participant in writing.

Data was collected by the researcher (audiologist) via verbatim documentation of respondents' answers and audiotaped recordings of interviews which were transcribed. Questions were also asked in numerical order to ensure consistency of format and organisation between interviews. Question explanation and probing was not applied beyond the methodological protocol in order to ensure consistency between interviews. To maintain consistency between interviews, digression from the interview script beyond pilot project alterations was avoided. Conducting consistent and structured interview sessions, as per the procedures delineated, ensured uniformity between and within interviews. Schiavetti and Metz (2002) state that consistency in methods to collect and evaluate data enhances a study's reliability. Consistency within the content analysis process is recommended in order to facilitate improved reliability of results obtained (Neuendorf, 2002).

Subject bias, such as the Hawthorne effect where participants heightened awareness that they form part of an evaluation process, may have influenced their performance. It must be acknowledged that participants are not passive partakers in the research process where they may potentially react to the content of the research as well as to the research process itself (Maxwell \& Satake, 2006). These factors are seen to have been reduced but only to some extent, by having verbally ensured participant confidentiality with regard to their responses, with the added assurance that their job security could not be affected in any way.

The time frame for administering the structured interviews spanned a maximum of one hour per interview.

\section{Data analysis and statistical procedures}

As the research design was specifically qualitative in nature, direct cause-effect relationships could not be inferred (Schiavetti \& Metz, 2002) from the attribute variables associated with the different socio-economic demographic 
variables of the provinces (North-West and Gauteng) from which the target participants were derived. No variables were manipulated and the researcher evaluated comparisons and contrasts derived from the data obtained from the sample groups located purposefully in different demographic contexts.

The data obtained was analysed using content analysis (Neuendorf, 2002) where transcriptions were evaluated to determine and code the emerging themes (Burns \& Grove, 2001; Neuendorf, 2002). Specifically, themes were not pre-determined but were words which were categorised according to common precepts and emergent themes identified. Quantitative data analyses, through the use of frequency calculations were condensed into tabular format for ease of frequency comparison between regions. This quantitative process was used as a precursor to the descriptive process, and this combination of processes was conducted as per the area of investigation. Comparisons and contrasts were evaluated between the two provincial sample groups. Specific comparisons were made as follows:

- The focus was on analysis of trends pertaining to the procedures and protocols of new-born and infant hearing screening conducted in each province, with the added possible influence of differing demographic attributes as classified according to the deprivational index based on the community survey (CS) 2007, where the North-West Province is considered to be more disadvantaged than the Gauteng Province (Day \& Gray, 2008).

- Questionnaire items addressed the specific procedures and protocol in use pertaining to new-born and infant hearing screening with the following areas of focus:

- Otoscope availability

- Otoscope usage

- Evaluative methods or instruments used other than otoscopic evaluation to assess risk for hearing loss

- Referrals emanating from evaluative methods or instruments used other than otoscopic evaluation to assess risk for hearing loss

- Other risk for hearing loss methods and the timing thereof, including (1) reviewing medical records, (2) interviewing the infant caregiver, and (3) physically examining the infant.

Content analysis was applied to all responses transcribed verbatim where frequencies of emergent themes were calculated. Further qualitative descriptive analyses were conducted in reference to the socio-economic demographics of the particular region in question positioned against its comparative counterpart as defined on the Community Survey deprivational index (Day \& Gray, 2008).

Limitations of the chosen method of analysis include non-generalisability of results to larger samples of the population under investigation due to the small sample size of participants. Results of smaller groups may not be representative of larger populations (Babbie, 1995; Maxwell \& Satake, 2006).

\section{Reliability and validity}

In order to ensure research reliability in the current research study, controls were exercised pertaining to participant variables, parameters pertaining to the questionnaire used to obtain information and the interview procedures employed. Over and above conducting site observations; utilising an independent rater during data analysis; a pilot study was also conducted to ensure reliability and validity in the current study.

In order to validate the questionnaire and protocol used, a pilot study was conducted prior to the main research project. Pre-test administration of the research instrument on three nursing immunisation clinic managers that shared target population criteria was conducted and this allowed the researcher to determine validity and reliability by identifying flaws in the research process, for example ambiguous questions and statements, leading questions, biased questions, timeframes associated with the interview process, and researcher bias (Maxwell \& Satake, 2006). All methodological processes and procedures followed were those of the main study. These pilot study participants were not included in the sample for the main study.

\section{Results Otoscope availability}

All MCWH primary health care clinics (PHCs) had otoscopes readily available at their clinics, with one otoscope dedicated to each consultation room; with at least one spare per clinic. Twenty four of the thirty respondents $(80 \% ; N=30)$ reported these to be working, with the rest having commented that they were unsure whether all were in working order. Of the respondents unsure of the working condition of the otoscope, there was an equal distribution between both provinces.

\section{Otoscope usage}

As a first step in the Integrated Management of Childhood Illnesses (IMCI) ear screening process, it was considered important to understand any variance in otoscope usage application. As depicted in Figure 1, the majority of respondents $(76.7 \% ; N=30)$ stated that they only conduct otoscopic examinations on some babies, with only two respondents (one from Gauteng [GP-COJ], and one from North-West [NW-M]) having stated that they do not conduct otoscopic evaluations on any babies under the age of five years. This is reflected in Figure 1.

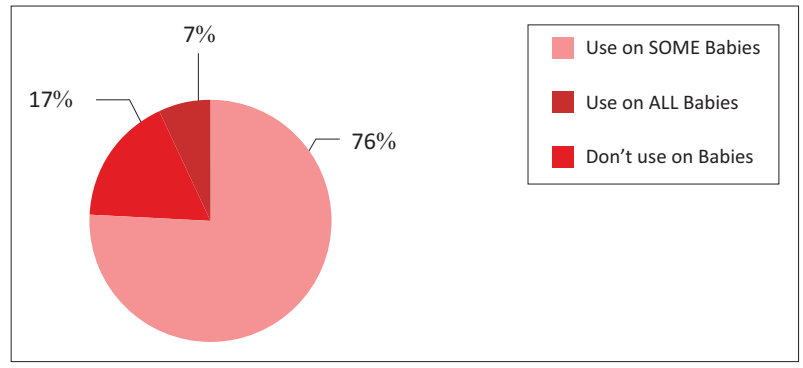

FIGURE 1: Otoscope usage on children below 5 years of age. 
TABLE 1: Themes identified regarding otoscope usage on some babies $(N=23)$.

\begin{tabular}{|c|c|c|c|c|c|c|c|c|c|c|}
\hline \multirow[t]{3}{*}{ Region } & \multicolumn{10}{|c|}{ Otoscope usage on some babies - Themes identified } \\
\hline & \multicolumn{2}{|c|}{$\begin{array}{l}\text { At Road to Health } \\
\text { Chart (RtHC) } \\
\text { milestone } \\
\text { age }\end{array}$} & \multicolumn{2}{|c|}{$\begin{array}{l}\text { On babies with } \\
\text { ear-related } \\
\text { problems } \\
\text { (unspecified) } \\
\end{array}$} & \multicolumn{2}{|c|}{$\begin{array}{c}\text { On babies with ear- } \\
\text { related problems } \\
\text { as reported by the } \\
\text { caregiver } \\
\end{array}$} & \multicolumn{2}{|c|}{$\begin{array}{c}\text { On babies with ear- } \\
\text { related Upper Respiratory } \\
\text { Tract Infection (URTI) } \\
\text { problems } \\
\end{array}$} & \multicolumn{2}{|c|}{$\begin{array}{c}\text { On babies with } \\
\text { ear-related child } \\
\text { behaviour } \\
\text { problems }\end{array}$} \\
\hline & $n$ & $\%$ & $n$ & $\%$ & $n$ & $\%$ & $n$ & $\%$ & $n$ & $\%$ \\
\hline Gauteng Province - City of Johannesburg District $(n=4)$ & 1 & & 1 & & 2 & & 3 & & 1 & \\
\hline Gauteng Province-Randfontein/Mogale District $(n=7)$ & 1 & & 1 & & 2 & & 5 & & 1 & \\
\hline Total Gauteng Province $(n=11)$ & 2 & $18.8 \%$ & 2 & $18.8 \%$ & 4 & $36.4 \%$ & 8 & $72.7 \%$ & 2 & $18.8 \%$ \\
\hline North-West Province - Merafong District $(n=6)$ & 0 & & 2 & & 2 & & 5 & & 0 & \\
\hline North-West Province - Potchefstroom District $(n=6)$ & 1 & & 4 & & 3 & & 3 & & 2 & \\
\hline Total North-West Province $(n=12)$ & 1 & $8.3 \%$ & 6 & $50.0 \%$ & 5 & $41.7 \%$ & 8 & $66.7 \%$ & 2 & $16.7 \%$ \\
\hline Total Gauteng Province + North-West Province $(N=23)$ & 3 & $13.0 \%$ & 8 & $34.8 \%$ & 9 & $39.1 \%$ & 16 & $69.6 \%$ & 4 & $17.4 \%$ \\
\hline
\end{tabular}

Note: As respondents may not have elaborated spontaneously, or may have offered more than one alternative, frequency calculations may not be equal to $n$.

Key: Under discussion

Otoscope usage on some babies was only slightly more prevalent in NW-P than in GP, with 11 GP respondents $(73.3 \% ; N=15)$ and $12 \mathrm{NW}$ respondents $(80 \%, N=15)$ doing so. Twenty three respondents elaborated on when and why they only evaluated some babies. These were documented and categorised into themes as reflected in Table 1.

As reflected in Table 1, the identified themes revealed that the majority $(69.9 \%)$ of otoscopic examinations was performed in accordance with infants' presentation of upper respiratory tract-related symptoms. NW-M district was the only region not to conduct otoscopic examinations on children exhibiting ear-related discomfort problems or at DoH (2004) RtHC milestone ages. Such discomfort was specified as ear scratching and/or pulling at ears, and/or crying when ears were touched. In addition, it was predominantly the NW-P region that conducted otoscopic examination on babies that presented with ear-related problems, although the nature of these problems remained unspecified. However, NW-P respondents $(3 ; n=6)$ demonstrated only a $50 \%$ confirmation that otoscopic examination was conducted on infants presenting with upper respiratory tract infection (URTI) symptoms. In fact, two NW-P respondents claimed that the IMCI code of conduct only warranted otoscopic evaluation to investigate ear obstruction and ear drainage post medication to assess treatment efficacy. No documentation in support of these claims was accessible to the researcher.

\section{Evaluative methods/instruments used other than otoscopic evaluation to assess risk for hearing loss}

A key element in the EHDI process is high yield from identification protocols at the earliest age possible (JCIH 2000; JCIHS, 2007; HPCSA, 2007). It was thus important not only to evaluate on which babies risk for hearing loss procedures were employed, but when these procedures were administered.

Only one respondent, located within the NW-M district, reported to not actively have used methods other than otoscopic evaluation on infants. Predominant themes were identified from 29 interviewees $(96.3 \% ; N=30)$ who reported to conduct evaluative methods other than otoscopic examination to assess risk for hearing loss. These included reviewing medical records (named as patient referral letters and RtHC running notes), interviewing caregivers, and conducting examinations of infant responses to environmental sounds incorporating noisemaker and/or verbal stimuli. All respondents reported that no formalised hearing screening was conducted at their clinics, as they did not have the equipment or know-how to do so. Budgetary and human resource constraints with no staff training were the main reasons cited.

\section{Environmental sounds incorporating noisemaker and/or verbal stimuli $(n=29)$}

Twenty two respondents $(75.9 \% ; N=29)$ revealed that they conducted a hearing screening comprised of presenting environmental sounds to the infant whilst monitoring the infant's behavioural responses, and 11 respondents (37.9\%; $N=29$ ) also commented that they evaluated the infant's behavioural responses to speech. These were reported to be conducted at different times, not necessarily on all babies but either when an ear related problem was indicated or at RtHC milestone assessments. At RtHC immunisations or milestones review, physically examining babies' responses to noisemakers and/or speech stimuli was conducted by $30 \%$ $(N=30)$ of respondents (6 GP and $3 \mathrm{NW}$ respondents).

The techniques used to screen infants' behavioural responses to environmental and speech sounds were described as elementary, with environmental sound presentation such as shaking a rattle, knocking the tip of a pen on an object, banging a door, and talking to the baby. Some respondents detailed their stimuli (noisemakers and speech) as being presented loudly from behind the baby, whilst others indicated that this was not always the case, for example where presentation may have occurred within the child's visual field.

\section{Reviewing medical records and conducting caregiver interviews $(\boldsymbol{N}=\mathbf{3 0})$}

Reviewing medical records when a problem was indicated was conducted mostly $(76.7 \% ; N=30 ; \mathrm{BY} 13 \mathrm{NW}$ and $10 \mathrm{GP}$ respondents) on babies where a problem was indicated. Similarly, just over half $(53.3 \% ; N=30)$ stated they conducted caregiver interviews. At RtHC immunisations or milestones review, reviewing medical records was conducted by $63.3 \%$ ( $N=30$; by $11 \mathrm{NW}$ and 8 GP respondents) of respondents, interviewing the caregivers was performed by $16.7 \%(N=30)$ 
of respondents and physically examining babies' responses to noisemakers and/or speech stimuli was conducted by $30 \%$ $(\mathrm{N}=30)$ of respondents (6 GP and $3 \mathrm{NW}$ respondents).

Regarding all of the above screening processes, what is of concern is that only $20 \%(N=30)$ of respondents reported to review all babies' medical records, $43.3 \%(N=30)$ reported to interview all caregivers, and $26.7 \%(N=30$; by 4 NW-P and 2 GP-COJ respondents) stated they performed physical examinations of the babies' responses to noisemakers and/or speech stimuli on all babies.

\section{Referrals emanating from evaluative methods/ instruments used to assess risk for hearing loss}

Twenty five of the 30 respondents $(83.3 \% ; N=30 ; 13$ GP and $12 \mathrm{NW}$ ) made referrals to the district's tertiary area specialist as soon as an infant was suspected of having a hearing impairment based on the various assessment techniques in current use. Of the five remaining respondents $(16.7 \%$; $N=30$ ), two NW-M reported that referrals were made at the immediate second clinic assessment or parental report of possible negative hearing repercussions. Three of these remaining five respondents (2 GP-COJ clinics, 1 NW-M clinic), reported a six month delay in referral for further evaluation. Included in the IMCI otitis media and associated hearing loss protocol is the generalised reference to the importance of referrals of 'all patients with speech, language and/or auditory perceptual problems' (DoH, 2005, p. 18).

The aforementioned referrals of babies suspected of presenting with hearing loss were made either directly by the PHC nurse or the PHC facility doctor. The two sub-districts considered alike on the deprivational index (GP-COJ and NW-M; GP-R/M and NW-P) (Day \& Gray, 2008) performed similarly in terms of immediacy of referrals, with the two sub-districts deemed to be more advanced (GP-R/M and NW-P), demonstrating improved referrals as soon as a possible a hearing problem was detected. However, differences between the provinces were marked in reference to referral options, where GP demonstrated markedly more referrals $(53.3 \% ; n=15)$ to regional audiologists or tertiary level Speech Therapy and Audiology Departments compared to only $2(13.3 \% ; n=15)$ references made by NW participants to either a sub-district school nurse or an audiologist. Neither districts made mention of use of a high risk register for referral of infants hearing assessment.

\section{Discussion}

Results from the current study yielded results which are important to consider in efforts to implement EHDI in primary health care. With regard to otoscope availability, the Primary Health Care Package stipulates that otoscopes with spare batteries and bulbs is the basic equipment requirement in its Integrated Management of Childhood Illnesses (IMCI) protocol, as part of its norms and standards pertaining to prevention of hearing loss due to otitis media ( $\mathrm{DoH}, 2001$; 2005). Thus, the adequate supply of working otoscopes found in the current sample was anticipated across regions.
The above results cite URTI as a seemingly important marker of possible negative ear sequelae for PHC health care providers. As otitis media with effusion is a well-established complication of URTI (Skoner, 2000), it is not surprising that URTI is reported as the predominant alert to earrelated health issues for PHC health care providers, and is so defined by the DoH IMCI protocols and standards $(\mathrm{DoH}$, 2001; 2005). However, these primary health care Package IMCI standards specify the use of an otoscope to inspect the external auditory canal and tympanic membrane in specific reference to the detection of otitis media and otitis externa (DoH, 2005). These IMCI protocols also call for caregiver interviews pertaining to child behaviour in relation to ear discomfort and hearing (DoH, 2001; 2005). One would thus have anticipated otoscope usage in diagnosis and treatment of infants to be more prevalent than what was evident in the current primary health care setting. The overall impression was one of inconsistent application of otoscopic evaluation, where the IMCI intention is for more vigilant use thereof in the diagnosis of otitis media with effusion and monitoring of its treatment efficacy (DoH, 2005). The observation of compliance gaps within and across sub-districts may be attributable to the relative autonomy now exercised by districts pertaining to health care management ( $\mathrm{DoH}, 2009)$.

Furthermore, when comparing environmental sound stimulation techniques to the more standardised physiological audiological approaches for infant hearing screening, it becomes evident that these behavioural techniques require serious review since they have been proven to lead to false negatives, with their associated reduced sensitivity and specificity (Lutman, 2000).

From a clinical perspective, across districts, the behavioural techniques and procedures to screen risk for hearing loss found in the current study are in agreement with IMCI protocols, particularly as they relate to identification and treatment of otitis media in infants (DoH, 2001; 2005), as well as RtHC milestone hearing screenings ( $\mathrm{DoH}, 2004)$. However, it is the IMCI otitis media protocols ( $\mathrm{DoH}, 2001$; 2005) that have been adhered to more consistently with regard to assessment of risk for hearing loss within the PHC setting. The broader approach to assess for conditions other than otitis media has demonstrated less IMCI protocol adherence, with more inconsistency reported in assessing risk for hearing loss at RtHC milestone age (DoH, 2004). Lack of consensus regarding the uniform application of techniques to assess for risk of hearing loss marginalises those children who don't present with a history of ear-related issues through medical record review, caregiver reporting, or the more obvious presentation of an ear-related problem. The silent epidemic of hearing loss, where its effects, particularly in less severe presentation, may not be obvious to the observer (Swanepoel, Hugo \& Louw, 2005), necessitates active pursuit in identifying and diagnosing hearing loss as is recommended in both developed and developing world contexts (JCIHS, 2007; HPCSA, 2007). In addition, the methods advocated by the JCIHS (2007) and HPCSA (2007) for detection of hearing loss are recognised as being more reliable and accurate, 
inclusive of inherent greater sensitivity and specificity than the rudimentary processes currently described to be in use in the PHC setting included in the current study. This is cause for concern according to UNHS goals, where targeted yield, through use of a formalised programme with objective physiological measures, is at least $95 \%$ of the population at large (HPCSA, 2007; JCIHS, 2007). This has significant clinical implications for the need to render an improvement in hearing screening services to infants located within these districts.

Such improvements may be actualised by revisiting IMCI protocols, where inclusion of other etiologies of hearing loss are patently expressed in documentation and training processes, with less emphasis on otitis media and its possible hearing impairment consequences to the point of exclusion of other etiologies (DoH, 2005). In addition, greater emphasis must also be made of the RtHC hearing assessment protocol where hearing assessments are to be conducted routinely at 3 months, 6 months and over a year of age. In conjunction herewith, the local district authority's role in the variation of application of IMCI and RtHC protocols must be incorporated, especially considering the greater sense of autonomy that districts are now able to exercise ( $\mathrm{DoH}, 2009)$.

Exploration of themes pertaining to the nature and timing of referrals made is paramount to understanding the diagnostic support available, should a hearing deficit be suspected. Diagnostic follow-up is a critical ethical marker in the hearing screening process, where diagnostic services are propagated as inherent in any comprehensive and ethical hearing screening process (JCIH, 2000; JCIHS, 2007; HPCSA, 2007).

The high referral rate to district tertiary area specialists is encouraging as a first step to early identification and intervention of hearing loss, which is paramount to improving communication outcomes (Northern \& Downs, 1991), and associated literacy development and educational outcomes for those with hearing loss (DesJardin, Ambrose, Martinez \& Eisenberg, 2009).

With regard to the timing of referral for further investigation, the immediacy of such referrals is not specified, and given that symptoms in infants are not easily observable, and where false negatives are generally increased through subjective behavioural observation techniques (Lutman, 2000), one may anticipate marked referral delays within the PHC system when compared to the systems where more precise objective audiological techniques are used. Late referrals for hearing loss are in direct conflict with JCIH and HPCSA (JCIH, 2000; JCIHS, 2007; HPCSA, 2007) recommended guidelines, and are considered problematic because reduced timeous intervention renders hearing loss a severe threat to essential quality of life indicators (Swanepoel, Delport \& Swart, 2007).

In addition, this overall reduced referral rate and use of audiology services may highlight lack of respondent knowledge of services rendered by audiologists and/or may reflect on the regional and district policy. Again, closer examination of policy pertaining to district referrals of infants with hearing loss with consideration of the possible relative district autonomy (now permitted and considered as strategic by the DoH (2009) is required to adequately draw conclusions. This is recommended for future research endeavours.

\section{Conclusion}

The current study revealed results which highlight the importance of establishing existing protocols in use; reviewing their implementation as well as their effectiveness before national plans are recommended; which might be challenging to implement due to barriers that might exist on the ground. The fact that findings in the current study indicated that none of the clinics offered or provided formalised new-born or infant hearing screening and none actually even had equipment to do so; indicates the urgent need for systematic planning at the various levels of health care service delivery in this country to ensure successful implementation of EHDI. Deprivational index did not appear to influence current findings; which has implications for forward planning in PHC. The fact that budgetary and human resource constraints were the main reasons given for the lack of formalised hearing screening; with staff training in particular being one of the main reasons, should be considered when planning is underway. It is of significant concern that even the non-formalised hearing screening protocols that are in place demonstrated inconsistencies in application across districts and that none complied with HPCSA (2007) clinic guidelines. This is over and above their lack of sensitivity and specificity. Current findings are limited by the sample size; and their focus on only two provinces; although care was taken to select provinces thought to be a fair representation of the South African context. Replication of this study nationally could yield results which are better generalisable.

\section{Acknowledgements}

\section{Competing interests}

The authors declare that they have no financial or personal relationship(s) that may have inappropriately influenced them in writing this article.

\section{Authors' contributions}

L.P-B. (University of the Witwatersrand) and K.K-S. (University of the Witwatersrand) contributed equally to the writing of this article.

\section{References}

Babbie, E. (1995). The practice of social research (7th edn.). San Diego, CA: Wadsworth. Burns, N., \& Grove, S. (2001). The practice of nursing research: Conduct, critique and utilisation (4th edn.). Philadelphia: W.B. Saunders Co.

Day, C., \& Gray, A. (2008). Indicators: Health and related indicators. In South African Health Review 2008. (Chapter 16). Durban, South Africa: The Health Systems Trust.

Department of Health (DoH). (2001). The primary health care package for South Africa: a set of norms and standards. Retrieved from http://ww.doh.gov.za/docs/ policy/norms/full-norms.html 
Department of Health (DoH). (2004). Health chart guidelines. Retrieved from http:// www.doh.gov.za/docs/factsheets/guidelines/health/healthchart.htm

Department of Health (DoH). (2005, December 1). Hearing guidelines. Retrieved from http://www.doh.gov.za/docs/factsheets/guidelines/hearing.pdf

Department of Health (DoH). (2009, March 10). Strategic plan 2009/10-2011/12. Retrieved from http://www.doh.gov.za/department/strategic\%20plan.html

DesJardin, J., Ambrose, S., Martinez, A., \& Eisenberg, L. (2009). Relationships between speech perception abilities and spoken language skills in young children with hearing loss. International Journal of Audiology, 48, 248-259. http://dx.doi. org/10.1080/14992020802607423

Friderichs, N., Swanepoel, D., \& Hall, J. (2012. Efficacy of a community-based hearing screening program utilizing existing clinic personnel in Western Cape, South Africa. International Jounal of Otorhinolaryngology, 76, 552-559. http://dx.doi. org/10.1016/j.ijporl.2012.01.015

Health Professions Council of South Africa (HPCSA). (2007). Early hearing detection and intervention programmes in South Africa position statement year 2007. Retrieved from http://www.hpcsa.co.za/hpcsa/UserFiles/Files/Speech\%20language $\% 20$ from $\% 20$ hearing/EHDI5position $\% 20$ statement $\% 20$ (HPCSA\%2007).pdf

Hutt, N., \& Rhodes, C. (2008). Post-natal hearing loss in universal neonatal hearing screening communities: Current limitations and future directions. Journa of Pediatrics and Child Health, 44, 87-91. http://dx.doi.org/10.1111/j.14401754.2007.01275.x

Joint Committee on Infant Hearing (JCIH). (2000). Year 2000 position statement: Principles and guidelines for early hearing detection and intervention programs. Pediatrics, 106(4), 798-817. http://dx.doi.org/10.1542/peds.106.4.798

Joint Committee on Infant Hearing Screening (JCIHS). (2007). Year 2007 position statement: principles and guidelines for early hearing detection and intervention programs. Pediatrics, 120, 898-921. http://dx.doi.org/10.1542/peds.2007-2333

Kanji, A., Khoza-Shangase, K., \& Ballot, D. (2010). Hearing screening follow-up return rate in a very low birth weight project: A retrospective record review. South African Journal of Communication Health, 4(4), 95-99.

Lutman, M.E. (2000). Techniques for neonatal hearing screening. Seminars in Hearing Screening, 21, 367-378. http://dx.doi.org/10.1055/s-2000-13465

Maxwell, D., \& Satake, E. (2006). Research and statistical methods in communication sciences and disorders. New York, NY: Thomson Delmar Learning.

Mehl, A., \& Thomson, V. (2002). The Colarado newborn hearing screening project, 1992-1999: on the threshold of effective population-based universal newborn hearing screening. Pediatrics, 109(1), E7. http://dx.doi.org/10.1542/peds.109.1.e7

Meyer, M., Swanepoel, D., Le Roux, T., \& Van der Linde, M. (2012). Early detection of infant hearing loss in the private health care sector of South Africa. Internationa Jounal of Otorhinolaryngology, 76, 698-703. http://dx.doi.org/10.1016/j ijporl.2012.02.023

Moeller, M.P. (2000). Early intervention and language development in children who are deaf and hard of hearing. Pediatrics, 106, 1-9. http://dx.doi.org/10.1542/ peds.106.3.e43

Northern, J. L., \& Downs, M.P. (1991). Hearing in children (4th edn.). Baltimore: Williams and Wilkins.

Northern, J., \& Downs, M. (2002). Hearing in children. In J. Northern, \& M. Downs, Hearing in Children (5th edn.). Baltimore: Lippincott Williams \& Wilkins

Neuendorf, K. (2002). The content analysis guidebook. London, England: Sage Publications, Inc.

Office of the Deputy President T.M. Mbeki. (1997). White paper on an integrated national disability strategy. Cape Town, South Africa: Rustica Press. Retrieved from http://www.polity.org.za/htmlgovdocs/white_papers/disability1. html?rebookmark=1

Office of the President, K. Motlanthe. (2009). S.A. yearbook 2008/2009 health chapter 12. In D. Burger (Ed.), S.A. Yearbook 2008/2009 (16th edn., pp. 310-331). Pretoria, South Africa: GCIS

Olusanya, B. (2000). Hearing impairment prevention in developing countries: making things happen. International Journal of Pediatric Otorhinolaryngology, 55, 167-171. http://dx.doi.org/10.1016/S0165-5876(00)00392-X
Olusanya, B. (2005). Can the world's infants with hearing loss wait?. Internationa Journal of Pediatric Otorhinolaryngology 69, 735-738. http://dx.doi. org/10.1016/j.ijporl.2005.03.024

Olusanya, B. (2007). Addressing the Global Neglect of Childhood Hearing Impairment in Developing Countries. PLoS Medicine, 626-630.

Olusanya, B., Luxon, L., \& Wirz, S. (2005). Screening for early childhood hearing loss in Nigeria. Journal of Medical Screening, 12, 115-118. http://dx.doi. org/10.1258/0969141054855274

Olusanya, B., \& Okolo, A. (2006). Early hearing detection at immunization clinics in developing countries. International Journal of Pediatric Otorhinolaryngology, 70 1495-1498. http://dx.doi.org/10.1016/j.ijporl.2006.04.002

Olusanya, B., Ruben, R., \& Parving, A. (2006). Reducing the burden of communication disorders in the developing world: an opportunity for the millenium development project. Journal of the American Medical Association, 296, 441-444. http://dx.doi. org/10.1001/jama.296.4.441

Scheepers, L., Swanepoel, D., \& Le Roux, T. (2014). Why parents refuse hearing screening and default on follow-up rescreening - A South African perspective. International Journal of Pediatric Otorhinolaryngology, 78, 652-658. http:// dx.doi.org/10.1016/j.ijporl.2014.01.026

Schiavetti, N., \& Metz, D. (2002). Evaluating research in communicative disorders. Boston, MA: Allyn and Bacon.

Skoner, D.P. (2000). Complications of Allergic Rhinitis. Journal of Allergic Clinical Immunology, 105(6), S605-S609. http://dx.doi.org/10.1067/mai.2000. 106150

Statistics South Africa. (2007). Community Survey 2007 (revised version). (Statistical release P30301). Pretoria: Statistics South Africa. Retrieved from http://www. statssa.gov.za/PublicationsHTML/P03112997/html/P030112007.html

Streefland, P. (2005). Public health care under pressure in sub-Saharan Africa. Health Policy, 71, 375-382. http://dx.doi.org/10.1016/j.healthpol.2004.06.002

Swanepoel, D., Delport, S., \& Swart, J. (2007). Equal opportunities for children with hearing loss by means of early identification. South African Family Practice, 49, 3. http://dx.doi.org/10.1080/20786204.2007.10873492

Swanepoel, D., Ebrahim, S., Joseph, A., \& Friedland, P. (2007). Newborn hearing screening in a South African private health care hospital. International Journal of Pediatric Otorhinolaryngology, 71(6), 881-887. http://dx.doi.org/10.1016/j. ijporl.2007.02.009

Swanepoel, D., Hugo, R., \& Louw, B. (2005). Infant hearing loss - silent epidemic of the developing world. Audiology Today, 17(4), 12-16.

Swanepoel, D., Hugo, R., \& Louw, B. (2006). Infant hearing screening at immunisation clinics in South Africa. International Journal of Pediatric Otorhinolaryngology, 70(7), 1241-1249. http://dx.doi.org/10.1016/j.ijporl.2006.01.002

Swanepoel, D., Louw, B., \& Hugo, R. (2007). A novel service delivery model for infant hearing screening in developing countries. International Journal of Audiology, 46, 321-327. http://dx.doi.org/10.1080/14992020601188583

Theunissen, M., \& Swanepoel, D. (2008). Early hearing detection and intervention services in the public health sector in South Africa. International Journal of Audiology, 47 (Supplement 1), S23-S29.

White, K. (2006). Early intervention for children with permanent hearing loss: Finishing the EHDI Revolution. The Volta Review, 106(3), 237-258.

World Bank. (2009). South Africa county brief. Retrieved from http://web.worldbank. org/WBSITE/EXTERNAL/COUNTRIES/AFRICAEXT/SOUTHAFRICAEXTN/0, menuPK: 368086 pagePK:141132 piPK:141107 theSitePK:368057,00.html

Yee-Arellano, H., Leal-Garza, F., \& Pauli-Muller, K. (2006). Universal newborn hearing screening in Mexico: Results of the first two years. Journal of ijporl.2006.06.008

Yoshinaga-Itano. (2004). Levels of evidence: universal newborn hearing screening (UNHS) and early hearing detection and intervention systems (EHDI). Journal of Communication Disorders, 37, 451-465. http://dx.doi.org/10.1016/j. jcomdis.2004.04.008 